OPEN ACCESS

Edited by:

Marjorie Hines Woollacott, University of Oregon, United States

Reviewed by:

Giovanni Messina

University of Foggia, Italy

Teresa Dianne Hawkes,

Brain Electrophysiology Laboratory,

United States

*Correspondence:

Yang Li

liy1017@yeah.net

tThese authors have contributed equally to this work

Specialty section: This article was submitted to Exercise Physiology,

a section of the journal

Frontiers in Physiology

Received: 26 May 2018

Accepted: 26 April 2019

Published: 29 May 2019

Citation:

Li Y, Zhang H and Wang Y (2019) Tai Chi Ameliorates Coronary Heart Disease by Affecting Serum Levels

of miR-24 and miR-155.

Front. Physiol. 10:587.

doi: 10.3389/fphys.2019.00587

\section{Tai Chi Ameliorates Coronary Heart Disease by Affecting Serum Levels of miR-24 and miR-155}

\author{
Yang Li*t, Haiyang Zhang ${ }^{\dagger}$ and Yushi Wang \\ Department of Cardiovascular Center, The First Bethune Hospital of Jilin University, Changchun, China
}

The protective role of Tai Chi in coronary heart disease (CHD) has been widely reported. However, the exact molecular mechanism remains unclear. Serum levels of miR-24 and miR-155 have been found to potentially be involved with CHD risk. Thus, the effects of Tai Chi on CHD risk were explored by measuring serum levels of miR-24 and miR-155. A total of $326 \mathrm{CHD}$ patients were evenly divided into the Tai Chi (TG) and control (CG) groups. The activities of daily living ability (ADL) and exercise of selfcare agency (ESCA) scores were compared between the two groups. Left ventricular ejection fraction (LVEF), SF-36 life quality, self-rating anxiety scale (SAS) and self-rating depression scale (SDS) were used to evaluate subjects' cardiac function, quality of life, anxiety, and depression. Serum levels of miR-24 and miR-155 were measured by a real-time quantitative polymerase chain reaction (RT-qPCR). After a 6-month Tai Chi intervention, the ESCA, ADL, LVEF, and SF-36 scores in the TG group were higher than those in the CG group $(p<0.05)$. The time of arrhythmia and atrioventricular block recovery and hospital stay, and the scores of SAS and SDS in the TG group were lower than in the CG group ( $p<0.05)$. Serum levels of miR-24 and miR-155 in the TG group were also lower than in the CG group ( $p<0.05)$. In addition, serum levels of miR-24 and miR-155 were negatively associated with the ESCA, ADL, LVEF and SF-36 scores, and had adverse effects on life quality. Altogether, these present findings demonstrate that Tai Chi improves CHD prognosis, by affecting serum levels of the miR-24 and miR-155.

Keywords: Tai Chi, self-care ability, coronary heart disease, cardiac function, serum microRNA

\section{INTRODUCTION}

Cardiovascular disease is the leading cause of death and disability worldwide (Santulli, 2013). The number of coronary heart disease (CHD) patients continues to increase year on year (Fukumoto et al., 2017) and reducing the mortality rate has become an urgent social concern (Virtanen et al., 2017). Tai Chi is an ancient practice used to improve cardiovascular health. It improves aerobic endurance and psychosocial well-being and may be a promising exercise option in cardiac rehabilitation. With the improvement of nursing practices, Tai Chi has become a standard method for CHD care (Salmoirago-Blotcher et al., 2017; Liu et al., 2018). Tai Chi is a comprehensive and long-term program designed to reduce the physical and psychological impact of heart disease on patients, and improves their social psychology (Blake and Hawley, 2012; Zheng et al., 2014, 2015). 
However, the molecular mechanism employed in the protective role of Tai Chi on CHD progression, remains widely unknown. miRNAs are short non-coding RNAs that affect gene expression by binding to the $3^{\prime}$-UTR of target mRNAs and inhibit protein production via the destabilization of mRNA and translational silence. They can also target promoter regions and affect gene transcription (Cannell et al., 2008). microRNAs and their target genes may be involved in heart disease ( $\mathrm{Lu}$ et al., 2018). Serum levels of miR-24 have been found to be associated with heart failure mortality (Zhang et al., 2018). Serum miR-155 was reported as a biomarker for CHD diagnosis (Jia et al., 2017). SCN5A gene encodes voltage-gated $\mathrm{Na}^{+}$channel NaV1.5, which depolarizes cardiac action potential. Mutant SCN5A is involved in heart failure-related sudden cardiac death, and miR-24 suppresses SCN5A expression (Zhang et al., 2018). Angiotensin receptor type 1 (AT1R) polymorphism has been found to be associated with CHD risk (Buraczyńska et al., 2003; Watkins et al., 2006), and miR-155 regulates AT1R expression (Blanco et al., 2012). Exercise can reduce the expression of miR-155 in an animal model (Rocha et al., 2018), but no report for miR-24 has been published yet. Both miRNAs regulate the transcripts of the brain-derived neurotrophic factor (BDNF) (Zhao and Srivastava, 2007; Xu et al., 2017), which is related to exercise effects in the human body as well as depression (Lu et al., 2014).

Serum miR-24 and miR-155 can be a potential target for CHD therapy. Tai Chi may improve CHD symptoms by affecting serum levels of miR-24 and miR-155. Therefore, we explored the effects of Tai Chi on CHD patients, by analyzing serum levels of miR24 and miR-155.

\section{MATERIALS AND METHODS}

\section{Participants}

This study was approved by the Ethics Committee of The First Hospital of Jilin University Bethune (Changchun, China) and written informed consent was obtained from each participant before data collection. All participants were diagnosed with CHD. From March 2014 to March 2016, 1788 patients were admitted to the critical care unit of our hospital's cardiology department.

\section{Inclusion Criteria}

The patients were diagnosed with CHD according to the New York Heart Association (NYHA) standards (Hu et al., 2017). The patients had the following demographic characteristics: angiographically proven $\mathrm{CHD}$, heart function between I and III levels, LVEF $<40 \%$, and older than 18 years without dementia and an acute coronary event within the last 3 months. The spouse or family of patients served as caregivers, and they accompanied the patients at the time of enrollment.

\section{Exclusion Criteria}

The exclusion criteria were as follows: unclear patient awareness; the occurrence of shock and need for defibrillation to restore cardiac reflex; pregnant or lactating females; the
CHD risk associated with changes in systemic inflammation, such as rheumatoid arthritis and immunological deficiency; receiving medications including systemic steroids, non-steroidal

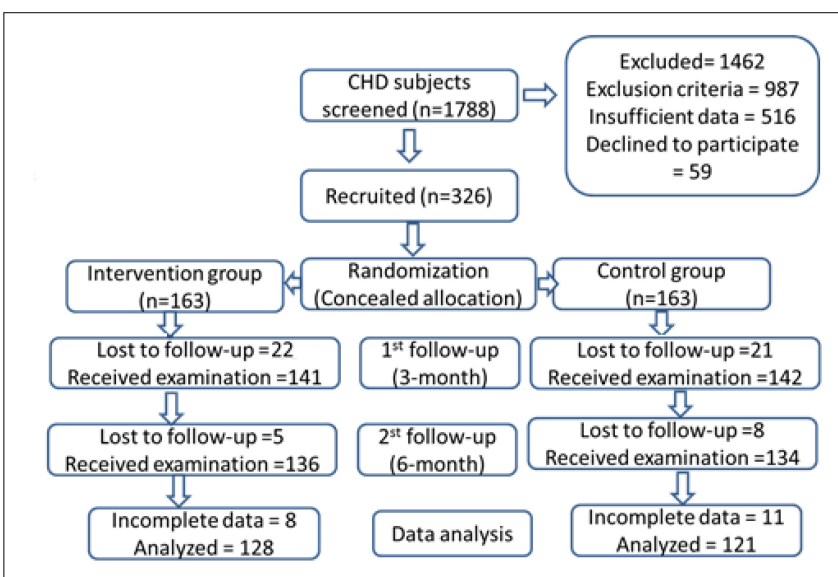

FIGURE 1 | Study flow diagram.

TABLE 1 | Clinical and demographic characteristics between two groups.

\begin{tabular}{|c|c|c|c|c|}
\hline & $\begin{array}{l}\text { Tai Chi } \\
\text { group } \\
(n=163)\end{array}$ & $\begin{array}{l}\text { Control } \\
\text { group } \\
(n=163)\end{array}$ & $f$-ratio & $p$ value \\
\hline Age (years) & $63.61 \pm 6.62$ & $65.44 \pm 5.79$ & 0.101 & 0.759 \\
\hline Gender, male (\%) & $72(44.2)$ & $68(41.7)$ & $x^{2}=0.200$ & 0.654 \\
\hline $\begin{array}{l}\text { BMl, weight/height }{ }^{2} \\
\left(\mathrm{~kg} / \mathrm{m}^{2}\right)\end{array}$ & $28.9 \pm 3.5$ & $29.4 \pm 3.7$ & 1.882 & 0.207 \\
\hline Smoking, cases (\%) & $26(16)$ & $28(17.2)$ & 0.089 & \\
\hline $\begin{array}{l}\text { Marital status, } \\
\text { married (\%) }\end{array}$ & $145(89.0)$ & $149(91.4)$ & 0.000 & 1.000 \\
\hline Monthly income, RMB & $4982 \pm 3256$ & $4618 \pm 3029$ & 2.456 & 0.127 \\
\hline Region & $\begin{array}{l}\text { Changchun } \\
\text { city }\end{array}$ & $\begin{array}{l}\text { Changchun } \\
\text { city }\end{array}$ & & \\
\hline Occupation, cases (\%) & & & 0.000 & 1.000 \\
\hline Unskilled, cases (\%) & $25(15.3)$ & $27(16.6)$ & & \\
\hline Professional, cases (\%) & $78(47.9)$ & $75(46)$ & & \\
\hline Skilled, cases (\%) & $60(36.8)$ & $61(37.4)$ & & \\
\hline Education & & & 0.000 & 1.000 \\
\hline Elementary, cases (\%) & $36(22.1)$ & $34(20.9)$ & & \\
\hline Middle school, cases (\%) & $30(18.4)$ & $32(19.6)$ & & \\
\hline $\begin{array}{l}\text { Professional school, } \\
\text { cases (\%) }\end{array}$ & $24(14.7)$ & $22(13.5)$ & & \\
\hline High school, cases (\%) & $43(26.4)$ & $42(25.8)$ & & \\
\hline $\begin{array}{l}\text { University degree, } \\
\text { cases (\%) }\end{array}$ & $30(18.4)$ & $33(20.2)$ & & \\
\hline NYHA class & & & 0.000 & 0.996 \\
\hline I, cases (\%) & $36(22.1)$ & 38 (23.3) & & \\
\hline II, cases (\%) & $49(30.1)$ & $58(35.6)$ & & \\
\hline III, cases (\%) & $77(47.2)$ & $67(41.1)$ & & \\
\hline IV, cases (\%) & 0 & 0 & & \\
\hline
\end{tabular}

Data are reported as means $\pm S D$ or number and percentages. BMI, body mass index; CHF, chronic heart failure; LVEF, left ventricular ejection fraction measurement; NYHA, New York Heart Association. The statistical test was performed with a chi-square test and multivariate analysis. 
TABLE 2 | Comparison of ESCA score and ADL score before and after nursing care between two groups.

\begin{tabular}{|c|c|c|c|c|c|c|}
\hline \multirow[t]{2}{*}{ Groups } & \multicolumn{3}{|c|}{ ESCA score } & \multicolumn{3}{|c|}{ ADL score } \\
\hline & 0 month & 3 months & 6 months & 0 month & 3 months & 6 months \\
\hline $\begin{array}{l}\text { Tai Chi } \\
\text { group }\end{array}$ & $86.6 \pm 11.7$ & $96.2 \pm 12.6$ & $122.5 \pm 13.4$ & $33.3 \pm 8.3$ & $38.2 \pm 8.9$ & $45.7 \pm 6.5$ \\
\hline $\begin{array}{l}\text { Control } \\
\text { group }\end{array}$ & $87.3 \pm 10.7$ & $90.3 \pm 11.8$ & $105.4 \pm 12.5$ & $32.0 \pm 7.6$ & $34.1 \pm 6.5$ & $39.6 \pm 4.8$ \\
\hline f-ratio & 0.146 & 0.458 & 2.964 & 0.247 & 2.53 & 2.76 \\
\hline$p$ & 0.412 & 0.359 & 0.00 & 0.352 & 0.038 & 0.007 \\
\hline
\end{tabular}

$n=128$ in Tai Chi group and $n=121$ in control group. The statistical test was performed with a multivariate analysis.

drugs, and hormone therapy and failing to comply with follow-up visits.

\section{Patients Grouping}

A total of 326 patients met the inclusion criteria and were enrolled in this study. Using a random number table, the subjects were evenly and randomly divided into the Tai Chi group (TG) and control group (CG). After a 6-month follow-up, 128 and 121 patients in TG and CG groups completed the present study, respectively (Figure 1).

\section{Tai Chi Intervention}

All patients were given conventional treatment and care, including the treatment of CHD symptoms, appropriate diets, exercises, medicine, and psychological therapy. The Tai Chi program consists of five movements and 24 forms in a Yang style (Verhagen et al., 2004). Patients in the TG group were trained by Tai Chi tutors. The classes lasted for $1 \mathrm{~h}$ per day, and the whole program was performed in the same place. In the CG group, the patients received physical exercise for $1 \mathrm{~h}$ per day according to previous reports (Aliabad et al., 2014).

\section{Exercise of Self-Care Ability Test (ESCA)}

Self-care ability was measured using a questionnaire provided by an instrument to measure ESCA according to a previous report (Kearney and Fleischer, 1979). The questionnaire includes four aspects: self-care skills (12 items), self-responsibility (eight items), self-concept (nine items), and health knowledge (14 items). The ESCA scores were divided into high (116-172), medium (58115), and low (0-57) levels.

TABLE 3 | Comparison of symptom improvement of CHD patients between two groups.

\begin{tabular}{lcccc}
\hline Groups & $\begin{array}{c}\text { Recovery time } \\
\text { of arrhythmia } \\
\text { (days) }\end{array}$ & LVEF\% & $\begin{array}{c}\text { Recovery time of } \\
\text { atrioventricular } \\
\text { block (days) }\end{array}$ & $\begin{array}{c}\text { Hospital } \\
\text { stay (days) }\end{array}$ \\
\hline Tai Chi group & $4.5 \pm 1.2$ & $47.3 \pm 3.4$ & $3.3 \pm 1.6$ & $16.4 \pm 2.5$ \\
Control group & $6.3 \pm 1.3$ & $42.6 \pm 3.1$ & $5.5 \pm 1.4$ & $20.5 \pm 4.8$ \\
$f$-ratio & 2.785 & 1.953 & 3.652 & 2.218 \\
$p$ & 0.015 & 0.043 & 0.006 & 0.032 \\
\hline$n=128$ in Tai Chi group and $n=121$ in control group. The statistical test was \\
performed with a multivariate analysis.
\end{tabular}

TABLE 4 | Comparison of the SF-36 life quality (a score between 0 and 100) between two groups.

\begin{tabular}{|c|c|c|c|}
\hline & $\begin{array}{c}\text { Tai Chi group, } \\
n=128\end{array}$ & $\begin{array}{c}\text { Control group, } \\
n=121\end{array}$ & $p$ values \\
\hline \multicolumn{4}{|l|}{0 month } \\
\hline PF & $33.9 \pm 6.9$ & $35.1 \pm 4.3$ & 0.712 \\
\hline $\mathrm{RP}$ & $32.7 \pm 5.3$ & $34.4 \pm 6.2$ & 0.342 \\
\hline $\mathrm{RE}$ & $26.2 \pm 6.3$ & $27.9 \pm 5.6$ & 0.885 \\
\hline SF & $42.5 \pm 4.1$ & $41.5 \pm 3.1$ & 0.873 \\
\hline $\mathrm{GH}$ & $56.9 \pm 5.8$ & $54.5 \pm 4.3$ & 0.801 \\
\hline $\mathrm{BP}$ & $37.6 \pm 6.7$ & $37.9 \pm 3.6$ & 0.718 \\
\hline $\mathrm{VT}$ & $35.9 \pm 3.7$ & $38.1 \pm 4.1$ & 0.523 \\
\hline $\mathrm{MH}$ & $45.4 \pm 5.3$ & $43.3 \pm 5.1$ & 0.429 \\
\hline Average & $39.5 \pm 5.8$ & $36.8 \pm 4.9$ & 0.278 \\
\hline \multicolumn{4}{|c|}{ 3-month follow-up } \\
\hline PF & $45.7 \pm 7.4$ & $38.9 \pm 5.8$ & 0.023 \\
\hline $\mathrm{RP}$ & $42.8 \pm 4.0$ & $35.6 \pm 5.1$ & 0.018 \\
\hline $\mathrm{RE}$ & $32.7 \pm 4.6$ & $28.1 \pm 6.5$ & 0.032 \\
\hline SF & $53.7 \pm 6.3$ & $40.1 \pm 5.2$ & 0.009 \\
\hline $\mathrm{GH}$ & $51.4 \pm 5.8$ & $47.3 \pm 3.5$ & 0.068 \\
\hline $\mathrm{BP}$ & $39.8 \pm 6.5$ & $38.1 \pm 4.0$ & 0.187 \\
\hline $\mathrm{VT}$ & $41.5 \pm 7.3$ & $37.2 \pm 5.5$ & 0.094 \\
\hline $\mathrm{MH}$ & $46.0 \pm 8.8$ & $44.3 \pm 6.7$ & 0.292 \\
\hline Average & $44.6 \pm 6.7^{*}$ & $39.1 \pm 4.3^{*}$ & 0.045 \\
\hline \multicolumn{4}{|c|}{ 6-month follow-up } \\
\hline PF & $62.1 \pm 10.8$ & $42.9 \pm 6.3$ & 0.001 \\
\hline $\mathrm{RP}$ & $65.8 \pm 3.9$ & $36.7 \pm 4.1$ & 0.001 \\
\hline $\mathrm{RE}$ & $61.2 \pm 5.7$ & $44.9 \pm 7.3$ & 0.001 \\
\hline SF & $63.4 \pm 7.4$ & $33.8 \pm 5.6$ & 0.001 \\
\hline $\mathrm{GH}$ & $62.1 \pm 6.3$ & $56.1 \pm 3.7$ & 0.014 \\
\hline $\mathrm{BP}$ & $54.8 \pm 7.2$ & $48.8 \pm 4.5$ & 0.001 \\
\hline VT & $66.7 \pm 8.5$ & $36.3 \pm 6.2$ & 0.001 \\
\hline $\mathrm{MH}$ & $48.1 \pm 9.6$ & $42.1 \pm 7.3$ & 0.042 \\
\hline Average & $61.5 \pm 7.4^{*}$ & $40.0 \pm 5.3^{*}$ & 0.001 \\
\hline
\end{tabular}

Follow-up was 3 months. SF-36 life quality includes $P F$, physiologic functioning; $R P$, role physical; BP, bodily pains; $G H$, general health; VT, vitality; SF, social functioning; $R E$, role of limitation due to emotional problems; and $\mathrm{MH}$, mental health. The statistical test was performed with a multivariate analysis.

\section{Assessment of Daily Living Ability (ADL)}

The ADLs were measured according to a previous report (Xiaolan, 2017). The scale was divided into two parts with 14 indicators, which were scored from 1 to 4 . The scale was scored 
as follows: 1, daily living activity could be performed; 2 , there were some difficulties when daily activities were performed; 3, help was needed when daily activities were performed and 4, daily activities could not be performed. Individuals who scored 1 were considered normal, and those who scored 4 to 6 were considered to have a decline in function or functional dependence. The scale score ranged from 1 to 56 . Scores $\leq 20$ indicated that the patients could take care of themselves, and scores $>20$ indicated that the patients could not take care of themselves. The homogeneity reliability was 0.84 and the CVI was 0.93 .

\section{Measurement of Cardiac Function}

LVEF was measured to evaluate the improvement of cardiac function. Transthoracic echocardiography was performed in all patients using Vivid E9 (GE Healthcare, Milwaukee, WI, United States). LVEF was calculated from the apical four- and two-chamber views using a Simpson's biplane method (Duncan et al., 2011). We investigated the time of arrhythmia and atrioventricular block recovery, and the hospitalization duration after nursing care.

\section{Measurement of Life Quality}

Life quality was measured according to eight aspects of SF-36 life quality: physiologic functioning (PF), role physical (RP), bodily pains $(\mathrm{BP})$, general health $(\mathrm{GH})$, vitality $(\mathrm{VT})$, social functioning (SF), role limitation due to emotional problems (RE), and mental health $(\mathrm{MH})$. These items were scored from 0 to 100 . The scores indicated the life quality.

\section{Anxiety and Depression Measurement}

The self-rating anxiety scale (SAS) and self-rating depression scale (SDS) are simple tools used to assess depression (Taylor et al., 2005) and emotional disturbance. Depression (Jiang et al., 2018) and poor emotional control (Potijk et al., 2016) are considered significant predictive risk factors for CHD patients. Moreover, depression may affect the recovery of CHD patients (Shen and Gau, 2017). A meta-analysis showed that exercise of continuous moderate-intensity significantly increases LVEF in patients with heart failure (Tucker et al., 2018). Exercise can reduce depressive and anxiety and improve outcomes of heart failure patients (Chiala et al., 2018).

The physical and psychological status (anxiety and depression) of patients in the CG and TG groups was investigated using SAS (Li et al., 2016) and SDS (Sahni and Agius, 2017) between CG and TG groups. The following criteria were considered using the scores: below 50, without depression/dysphoria; 50-59, mild depression/dysphoria; 60-69, moderate depression/dysphoria and above 70, with high depression/dysphoria.

\section{Measurement of Serum Levels of miR-24 and miR-155}

Five milliliters of blood was obtained from each subject via venipuncture, and the serum was isolated from the blood using centrifugation at $2000 \times g$ for $10 \mathrm{~min}$. Serum total RNA was extracted using a kit (Ambition, Life Technologies, Carlsbad, CA, United States) according to the manufacturer's

TABLE 5 | Comparison of SAS/SDS scores between groups.

\begin{tabular}{|c|c|c|c|c|c|c|}
\hline \multirow[t]{2}{*}{ Groups } & \multicolumn{3}{|c|}{ SAS } & \multicolumn{3}{|c|}{ SDS } \\
\hline & 0 month & 3 months & 6 months & 0 month & 3 months & 6 months \\
\hline $\mathrm{TG}$ & $55.6 \pm 4.6$ & $50.1 \pm 4.8$ & $32.3 \pm 4.5$ & $57.3 \pm 10.5$ & $48.3 \pm 9.9$ & $39.2 \pm 9.3$ \\
\hline CG & $53.9 \pm 6.9$ & $53.7 \pm 6.1$ & $43.9 \pm 5.6$ & $56.5 \pm 6.9$ & $54.1 \pm 7.0$ & $53.6 \pm 8.7$ \\
\hline$f$-ratio & 0.263 & 0.398 & 3.598 & 0.156 & 1.826 & 3.247 \\
\hline$P$ & 0.512 & 0.256 & 0.001 & 0.672 & 0.039 & 0.003 \\
\hline
\end{tabular}

SAS, self-rating anxiety scale; SDS, self-rating depression scale. The statistical test was performed with a multivariate analysis.

A

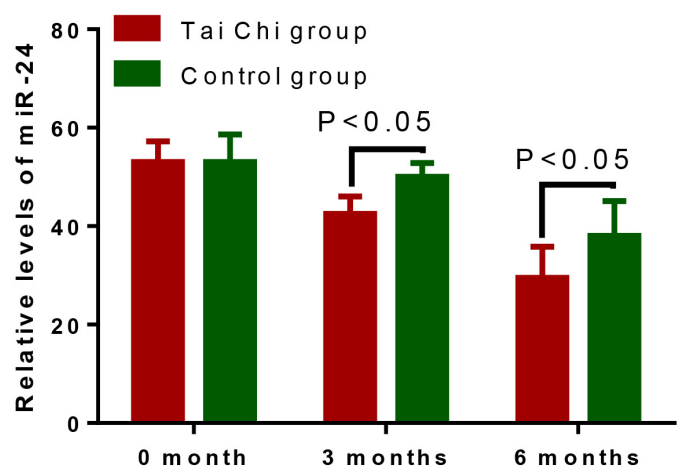

B

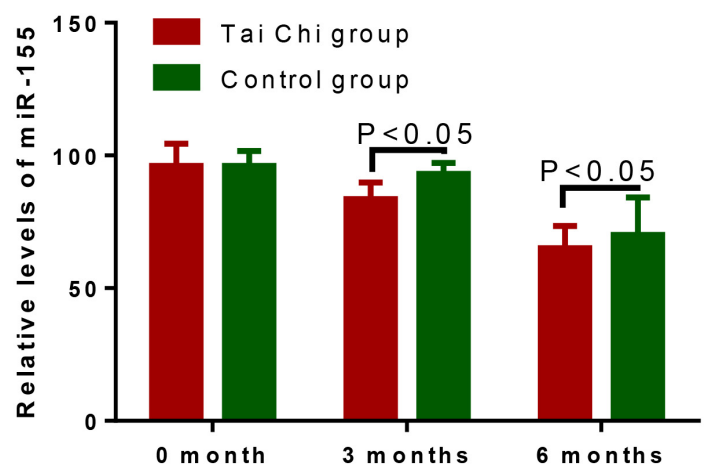

FIGURE 2 | The effects of Tai Chi on serum levels of miR-24 and miR-155. (A) the effects of Tai Chi on serum levels of miR-24. (B) the effects of Tai Chi on serum levels of miR-155. 

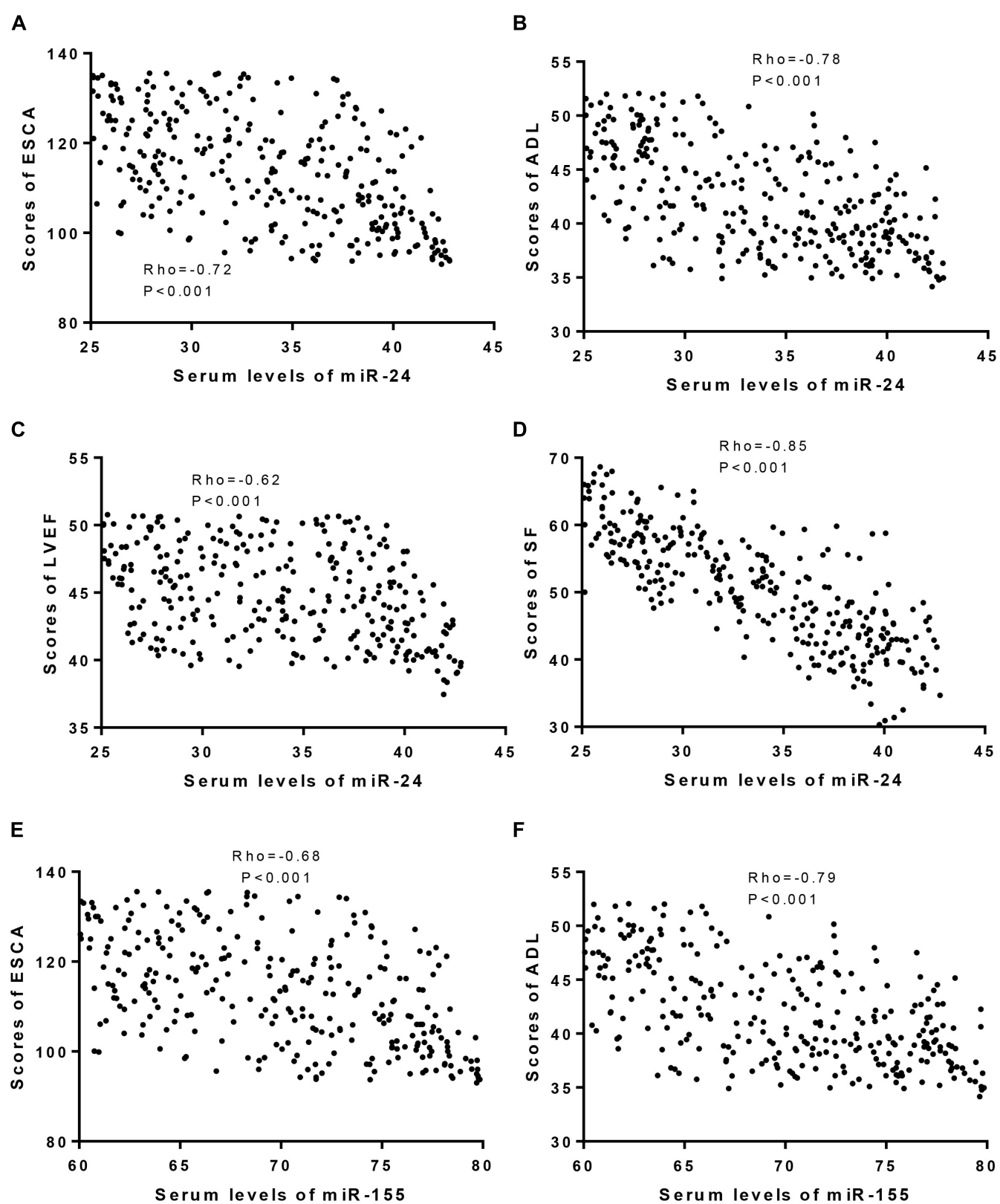

$\mathbf{F}$

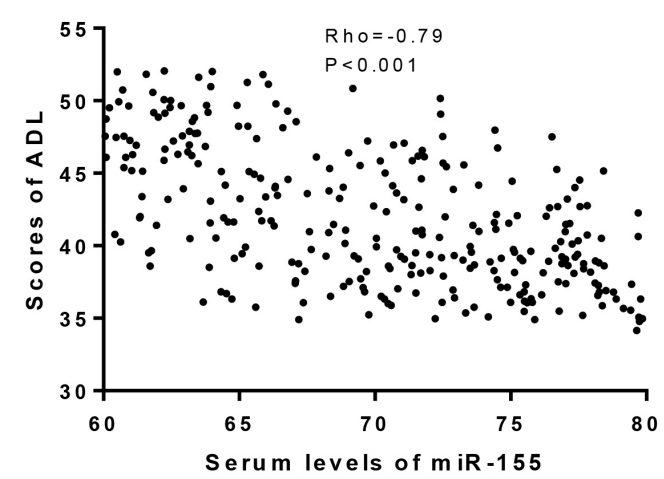

G
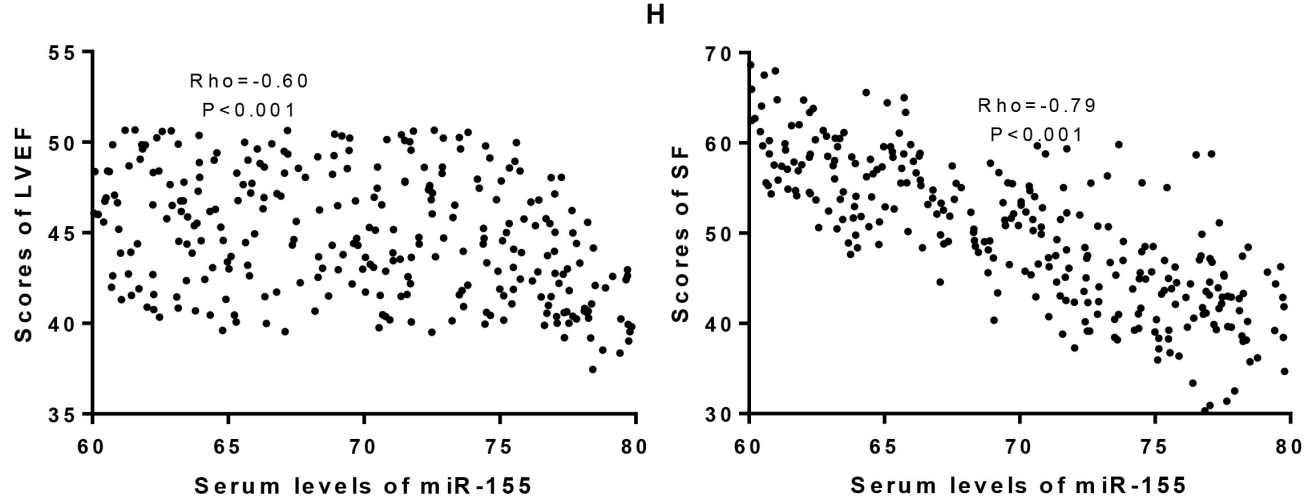

FIGURE 3 | Continued 
FIGURE 3 | An association test was used to test the coefficients between serum levels of miroRNA and scores of ESCA, ADL, LVEF and SF-36. (A) The relationship between serum levels of miR-24 and scores of ESCA. (B) The relationship between serum levels of miR-24 and scores of ADL. (C) The relationship between serum levels of miR-24 and scores of LVEF. (D) The relationship between serum levels of miR-24 and scores of SF-36. (E) The relationship between serum levels of miR-155 and scores of ESCA. (F) The relationship between serum levels of miR-155 and scores of ADL. (G) The relationship between serum levels of miR-155 and scores of LVEF. (H) The relationship between serum levels of miR-155 and scores of SF-36. There is a strong negative relationship if rho <-0.5.

instructions. Serum levels of miR-24 (forward primer: $5^{\prime}$ GTCGTATCCAGTGCAGGGTCCGAGGTATTCGCACTG-3'; reverse primer, 5'-GTGCAGGGTCCGAGGT-3'); and miR-155 (forward primer: $5^{\prime}$-GTCGTATCCAGTGCAGGGTCCGAGG$3^{\prime}$, reverse primer: $5^{\prime}$-TCGCACTGGATACGACCCCCTA-3') were measured by using real-time quantitative polymerase chain reaction (RT-qPCR). U6 was used as a control using snRNA-forward primer, CTCGCTTCGGCAGCACA, and snRNA-reverse primer, AACGCTTCACGAATTTGCGT.

\section{Statistical Analysis}

All data were presented as mean values \pm SD (standard deviations). A multivariate analysis of variance was used to assess the conditional effect of each variable (Hawkes et al., 2016a,b). It also reduced the number of statistical tests run on the same set of variables, thus controlling for alpha slippage. The statistical difference for the numbers (gender, smoking, and marital status) between the two groups was measured using the $\chi 2$ test. Other variables were analyzed using a multivariate analysis with adjusted hazard ratios and $95 \%$ confidence intervals. An association test was used to test the coefficients of two variants. All data were analyzed using SPSS 20.0 (SPSS Inc., United States). $p<0.05$ was considered statistically significant.

\section{RESULTS}

\section{Basic Clinical Characteristics}

Demographic variables included education, occupation, age, gender, BMI, monthly income, residence, NYHA class, and marital status. The average age of patients in the TG and CG groups were $62.6 \pm 5.62$ and $64.4 \pm 5.29$ years, respectively. Educational levels were similar in both groups. In addition, all patients in the two groups had a similar NYHA functional class, and no class IV was found. Clinical characteristics showed that a statistical difference was insignificant in the two groups (Table 1, $p>0.05$ ).

\section{Tai Chi Increased the ESCA and ADL Scores of CHD Patients}

After a 6-month follow-up, the ESCA and ADL scores in the TG group were $122.5 \pm 13.4$ and $45.7 \pm 6.5$, respectively, which were better than that in the CG group (105.4 \pm 12.5 and $39.6 \pm 4.8$, respectively) (Table 2, $p<0.05$ ).

\section{Tai Chi Improved the Symptoms of CHD Patients}

The recovery time of arrhythmia in the TG group was shorter than in the CG group (Table 3, $p<0.05$ ). LVEF was $47.3 \% \pm 3.4 \%$ in the TG group, which was higher than that in the CG group $(42.6 \% \pm 3.1 \%$, Table 3, $p<0.05)$; However, the time of atrioventricular block recovery and hospitalization duration in the TG group was lower than that in the CG group $(P<0.05)$. Tai Chi improved the clinical symptoms of CHD patients.

\section{Tai Chi Improved the SF-36 Life Quality Scores After Treatment}

Changes in the life quality scores were measured using SF36 scores before and after Tai Chi intervention. The average SF-36 scores in the TG and CG groups were $61.5 \pm 7.4$ and $44.0 \pm 5.3$ after a 6 -month follow-up, respectively. SF-36 life scores showed that the life quality of the TG group was improved in eight aspects, including PF, RP, BP, GH, VT, SF, $\mathrm{RE}$, and $\mathrm{MH}$. Comparatively, the patients in the CG group showed improvement in four aspects, including PF, RE, SF, and BP (Table 4).

\section{Tai Chi Improved Depression Symptoms After Treatment}

Before Tai Chi intervention, the statistical difference was insignificant between the two groups $(p>0.05$, Table 5). Compared with those in the CG group, the average values of SAS and SDS decreased significantly in the TG group, and the difference was statistically significant after a 6-month follow-up (Table 5, $p<0.05$ ). All these results demonstrated that Tai Chi improved depression after treatment.

\section{Tai Chi Reduced the Serum Levels of miR-24 and miR-155}

Before Tai Chi intervention, the statistical difference for serum miR-24 levels was insignificant (Figure 2A, $p>0.05$ ). After the 3and 6-month training, Tai Chi reduced the serum levels of miR-24 (Figure 2A, $p<0.05$ ). Similarly, before Tai Chi intervention, the statistical difference for serum miR-155 levels was insignificant (Figure 2B, $p>0.05$ ). After the 3- and 6-month intervention, Tai Chi reduced the serum levels of miR-155 (Figure 2B, $p>0.05$ ).

\section{Serum Levels of miR-24 and miR-155 Had Adverse Effects on Health-Related Quality of Life in CHD Patients}

An association test showed that serum miR-24 levels had a negative relationship with ESCA (Figure 3A), ADL (Figure 3B), LVEF (Figure 3C), and SF-36 (Figure 3D). Similarly, serum miR-155 also had a negative relationship with the ESCA (Figure 3E), ADL (Figure 3F), LVEF (Figure 3G), and SF-36 scores (Figure $3 \mathrm{H}$ ). The results suggested that serum miR-24 and miR-155 levels had adverse effects on the quality of life in CHD patients. 


\section{DISCUSSION}

Traditional Chinese exercises are very popular in China, especially Tai Chi, which is of great benefit to people's health and heart-related diseases. Tai Chi is suitable for any population as well as different ages. Tai Chi intervention is low risk and can be used to improve the quality of life for cardiovascular patients, especially CHD patients (Liu et al., 2018). The initial concept of Tai Chi is to make full use of available human resources and to motivate patients' initiative and to reduce the intensity of nurses' work. Depression and anxiety are well-known indicators of poor outcomes in CHD patients (Palacios et al., 2018). CHD patients undergoing percutaneous coronary intervention have a higher rate of depression than the general population. Treating depression has proven to be effective in improving outcomes of CHD patients (Feng et al., 2017).

In this study, anti-anxiety (Zheng et al., 2018) and antidepressant (Rawtaer et al., 2015; Siu et al., 2017) exercises of Tai Chi were applied to observe their impact on a patients self-care ability, ADLs, and disease prognosis. The results showed that ESCA, ADL and SF-36 scores in the TG group were significantly improved, and better than in the CG group (Table 2, $P<0.05$ ). Tai Chi intervention reduced the time of arrhythmia recovery and hospitalization duration. These results are consistent with previous reports. A Tai Chi exercise regimen can improve the physical function and ability of frail elderly individuals and reduce the demand for long-term care (Nomura et al., 2011). Tai Chi has been reported to ameliorate subsyndromal depression and anxiety in the elderly population (Rawtaer et al., 2015). Tai Chi is an effective strategy to enhance cognitive function and maintain movement abilities in patients with instrumental ADLs and mild cognitive impairment (Siu and Lee, 2018). Moreover, a previous study found that a 12-week Tai Chi intervention improved the LVEF in the patients with chronic kidney and cardiovascular diseases (Shi et al., 2014). Tai Chi has provided novel ideas and directions for the management of atrial fibrillation (the most common cardiac arrhythmia) management.

In the TG group, cardiac function was improved, and symptom recovery time was shortened $(P<0.05)$. The results demonstrated the value of Tai Chi in heart disease care. After intervention, patients became familiar with heart disease-related knowledge, strengthened their self-care skills, and enhanced their daily activities so as to improve their cardiac function and prognosis.

Serum miR-24 and miR-155 levels are associated with the risk of heart failure (Esmaeili-Bandboni et al., 2018; Zhang et al., 2018). The present work showed that Tai Chi intervention

\section{REFERENCES}

Aliabad, H. O., Vafaeinasab, M., Morowatisharifabad, M. A., Afshani, S. A., Firoozabadi, M. G., and Forouzannia, S. K. (2014). Maintenance of physical activity and exercise capacity after rehabilitation in coronary heart disease: a randomized controlled trial. Glob. J. Health Sci. 6, 198-208.

Blake, H., and Hawley, H. (2012). Effects of Tai Chi exercise on physical and psychological health of older people. Curr. Aging Sci. 5, 19-27. doi: 10.2174/ 1874609811205010019 reduced the serum levels of $\mathrm{miR}-24$ and $\mathrm{miR}-155$ in $\mathrm{CHD}$ patients (Figure 2, $p<0.05$ ). Furthermore, serum levels of miR-24 and miR-155 were negatively related with ESCA, ADL, LVEF and SF-36 scores (Figure 3, $p<0.05$ ). Tai Chi intervention improved $\mathrm{CHD}$ symptoms by reducing serum levels of miR24 and miR-155.

Tai Chi improved the self-care and living ability by increasing ESCA, ADL and SF-36 scores. It strengthened cardiac function by increasing the percentage of LVEF. In addition, Tai Chi reduced SAS and SDS scores significantly, and should be developed as a potential method in the prevention of CHD. Anxiety and depression are considered to have genetic roots (Petito et al., 2016), thus, Tai Chi may improve SAS and SDS scores by affecting the genes related to anxiety and depression.

Despite our findings, the present work has some limitations. First, Tai Chi could not be provided for most CHD patients because of the lack of well-trained Tai Chi tutors. Exploring the therapeutic effect of Tai Chi on CHD is important; Second, a molecular link between Tai Chi and prevention of $\mathrm{CHD}$ progression, will provide insights into the functional role of Tai Chi in CHD therapy; Third, different psychotherapists will have different skills, which might lead to different therapeutic results. A unified public platform for Tai Chi is highly demanded. Therefore, further work is still needed to address these important issues.

\section{CONCLUSION}

Tai Chi increased ESCA and ADL, LVEF and SF-36 scores, and significantly reduced SAS and SDS scores. Tai Chi improved the self-care, ADLs, cardiac function, and prognosis of CHD patients. Given these findings, Tai Chi should be developed as a potential method for CHD therapy.

\section{ETHICS STATEMENT}

This study was approved by the Ethics Committee of The First Hospital of Jilin University Bethune, and written informed consent was obtained from each adult before data collection. All participants were diagnosed with $\mathrm{CHD}$.

\section{AUTHOR CONTRIBUTIONS}

$\mathrm{HZ}$ and YW designed and performed the experiments. YW analyzed all data. YL wrote the manuscript.

Blanco, R. R., Austin, H., Vest, R. N. III, Valadri, R., Li, W., Lassegue, B., et al. (2012). Angiotensin receptor type 1 single nucleotide polymorphism 1166A/C is associated with malignant arrhythmias and altered circulating miR-155 levels in patients with chronic heart failure. J. Card. Fail. 18, 717-723. doi: 10.1016/j. cardfail.2012.06.531

Buraczyńska, M., Pijanowski, Z., Spasiewicz, D., Nowicka, T., Sodolski, T., Widomska-Czekajska, T., et al. (2003). Renin-angiotensin system gene polymorphisms: assessment of the risk of coronary heart disease. Kardiol. Pol. $58,5-8$. 
Cannell, I. G., Kong, Y. W., and Bushell, M. (2008). How do microRNAs regulate gene expression? Biochem. Soc. Trans. 36, 1224-1231. doi: 10.1042/BST0361224 Chiala, O., Vellone, E., Klompstra, L., Ortali, G. A., Stromberg, A., and Jaarsma, T. (2018). Relationships between exercise capacity and anxiety, depression, and cognition in patients with heart failure. Heart Lung 47, 465-470. doi: 10.1016/j.hrtlng.2018.07.010

Duncan, R. F., Dundon, B. K., Nelson, A. J., Pemberton, J., Williams, K., Worthley, M. I., et al. (2011). A study of the 16-segment regional wall motion scoring index and biplane simpson's rule for the calculation of left ventricular ejection fraction: a comparison with cardiac magnetic resonance imaging. Echocardiography 28, 597-604. doi: 10.1111/j.1540-8175.2011.01394.x

Esmaeili-Bandboni, A., Bagheri, J., Bakhshandeh, A. R., Mohammadnejad, J., and Sadroddiny, E. (2018). Serum levels of miR-155, miR-326, and miR-133b as early diagnostic biomarkers for the detection of human acute heart allograft rejection in comparison with serum cardiac troponin T. Heart Surg. Forum 21, E101-E107. doi: 10.1532/hsf.1887

Feng, C., Ji, T., Liu, Y., Chen, D., Dai, J., Ni, X., et al. (2017). Role of depression in secondary prevention of Chinese coronary heart disease patients receiving percutaneous coronary intervention. PLoS One 12:e0187016. doi: 10.1371/ journal.pone. 0187016

Fukumoto, R., Kawai, M., Minai, K., Ogawa, K., Yoshida, J., Inoue, Y., et al. (2017). Conflicting relationship between age-dependent disorders, valvular heart disease and coronary artery disease by covariance structure analysis: possible contribution of natriuretic peptide. PLoS One 12:e0181206. doi: 10. 1371/journal.pone.0181206

Hawkes, T. D., Bihl, T. J., and Woollacott, M. H. (2016a). "Interacting Systems Approach to Investigation of Human Cognitive Capacity" in Proceedings of the 2016 Cincy-Dayton INFORMS Conference. Cincinnati, OH. 33-40

Hawkes, T. D., Eveland, E., Bihl, T. J., Frey, J. S., and Mauzy, C. A. (2016b). Influence of BDNF Genotype and Exercise Type on Post-Training, Post-Acute Exercise Bout VO2Max and BDNF Serum levels. Technical Report 88ABW-2017-2399. Wright-Patterson AFB, OH: Air Force Research Laboratory.

Hu, Y., Jiang, S., Lu, S., Xu, R., Huang, Y., Zhao, Z., et al. (2017). Echocardiography and electrocardiography variables correlate with the New York heart association classification: an observational study of ischemic cardiomyopathy patients. Medicine 96:e7071. doi: 10.1097/MD.0000000000007071

Jia, Q. W., Chen, Z. H., Ding, X. Q., Liu, J. Y., Ge, P. C., An, F. H., et al. (2017). Predictive effects of circulating miR-221, miR-130a and miR-155 for coronary heart disease: a multi-ethnic study in China. Cell. Physiol. Biochem. 42, 808-823. doi: $10.1159 / 000478071$

Jiang, X., Asmaro, R., O’sullivan, D. M., Modi, J., Budnik, E., and Schnatz, P. F. (2018). Depression may be a risk factor for coronary heart disease in midlife women < 65years: a 9-year prospective cohort study. Int. J. Cardiol. 271, 8-12. doi: 10.1016/j.ijcard.2018.05.085

Kearney, B. Y., and Fleischer, B. J. (1979). Development of an instrument to measure exercise of self-care agency. Res. Nurs. Health 2, 25-34. doi: 10.1016/j. clnu.2013.03.018

Li, H., Jin, D., Qiao, F., Chen, J., and Gong, J. (2016). Relationship between the self-rating anxiety scale score and the success rate of 64-slice computed tomography coronary angiography. Int. J. Psychiatry Med. 51, 47-55. doi: 10. 1177/0091217415621265

Liu, T., Chan, A. W., Liu, Y. H., and Taylor-Piliae, R. E. (2018). Effects of Tai Chi-based cardiac rehabilitation on aerobic endurance, psychosocial well-being, and cardiovascular risk reduction among patients with coronary heart disease: a systematic review and meta-analysis. Eur. J. Cardiovasc. Nurs. 17, 368-383. doi: $10.1177 / 1474515117749592$

Lu, J., Xu, Y., Hu, W., Gao, Y., Ni, X., Sheng, H., et al. (2014). Exercise ameliorates depression-like behavior and increases hippocampal BDNF level in ovariectomized rats. Neurosci. Lett. 573, 13-18. doi: 10.1016/j.neulet.2014. 04.053

Lu, Q., Sun, Y., Duan, Y., Li, B., Xia, J., Yu, S., et al. (2018). Comprehensive microRNA profiling reveals potential augmentation of the IL1 pathway in rheumatic heart valve disease. BMC Cardiovasc. Disord. 18:53. doi: 10.1186/ s12872-018-0788-2

Nomura, T., Nagano, K., Takato, J., Ueki, S., Matsuzaki, Y., and Yasumura, S. (2011). The development of a Tai Chi exercise regimen for the prevention of conditions requiring long-term care in Japan. Arch. Gerontol. Geriatr. 52, E198-E203. doi: 10.1016/j.archger.2010.11.010
Palacios, J., Khondoker, M., Mann, A., Tylee, A., and Hotopf, M. (2018). Depression and anxiety symptom trajectories in coronary heart disease: associations with measures of disability and impact on 3-year health care costs. J. Psychosom. Res. 104, 1-8. doi: 10.1016/j.jpsychores.2017.10.015

Petito, A., Altamura, M., Iuso, S., Padalino, F. A., Sessa, F., D’andrea, G., et al. (2016). The Relationship between personality traits, the 5HTT polymorphisms, and the occurrence of anxiety and depressive symptoms in elite athletes. PLoS One 11:e0156601. doi: 10.1371/journal.pone.0156601

Potijk, M. R., Janszky, I., Reijneveld, S. A., and Falkstedt, D. (2016). Risk of coronary heart disease in men with poor emotional control: a prospective study. Psychosom. Med. 78, 60-67. doi: 10.1097/PSY.0000000000000254

Rawtaer, I., Mahendran, R., Yu, J., Fam, J., Feng, L., and Kua, E. H. (2015). Psychosocial interventions with art, music, Tai Chi and mindfulness for subsyndromal depression and anxiety in older adults: a naturalistic study in Singapore. Asia Pac. Psychiatry 7, 240-250. doi: 10.1111/appy.12201

Rocha, B., Rodrigues, A. R., Tomada, I., Martins, M. J., Guimaraes, J. T., Gouveia, A. M., et al. (2018). Energy restriction, exercise and atorvastatin treatment improve endothelial dysfunction and inhibit miRNA-155 in the erectile tissue of the aged rat. Nutr. Metab. 15:28. doi: 10.1186/s12986-018-0265-z

Sahni, A., and Agius, M. (2017). The Use of the PHQ9 self-rating scale to assess depression within primary care. Psychiatr. Danub. 29, 615-618.

Salmoirago-Blotcher, E., Wayne, P. M., Dunsiger, S., Krol, J., Breault, C., Bock, B. C., et al. (2017). Tai Chi Is a promising exercise option for patients with coronary heart disease declining cardiac rehabilitation. J. Am. Heart Assoc. 6:e006603. doi: 10.1161/JAHA.117.006603

Santulli, G. (2013). Epidemiology of cardiovascular disease in the 21st century: updated numbers and updated facts. J. Cardiovasc. Dis. 1, 1-2.

Shen, B. J., and Gau, J. T. (2017). Influence of depression and hostility on exercise tolerance and improvement in patients with coronary heart disease. Int. J. Behav. Med. 24, 312-320. doi: 10.1007/s12529-016-9598-z

Shi, Z. M., Wen, H. P., Liu, F. R., and Yao, C. X. (2014). The effects of tai chi on the renal and cardiac functions of patients with chronic kidney and cardiovascular diseases. J. Phys. Ther. Sci. 26, 1733-1736. doi: 10.1589/jpts.26.1733

Siu, K. C., Padilla, C., and Rajaram, S. S. (2017). The interrelationship between balance. Tai Chi and depression in Latino older adults. Aging Clin. Exp. Res. 29, 395-401. doi: 10.1007/s40520-016-0593-7

Siu, M. Y., and Lee, D. T. F. (2018). Effects of tai chi on cognition and instrumental activities of daily living in community dwelling older people with mild cognitive impairment. Bmc Geriatr. 18:37. doi: 10.1186/s12877-018-0720-8

Taylor, R., Lovibond, P. F., Nicholas, M. K., Cayley, C., and Wilson, P. H. (2005). The utility of somatic items in the assessment of depression in patients with chronic pain: a comparison of the zung self-rating depression scale and the depression anxiety stress scales in chronic pain and clinical and community samples. Clin. J. Pain 21, 91-100. doi: 10.1097/00002508-200501000-00011

Tucker, W. J., Beaudry, R. I., Liang, Y., Clark, A. M., Tomczak, C. R., Nelson, M. D., et al. (2018). Meta-analysis of exercise training on left ventricular ejection fraction in heart failure with reduced ejection fraction: a 10-year Update. Prog. Cardiovasc. Dis. 62, 163-171. doi: 10.1016/j.pcad.2018.08.006

Verhagen, A. P., Immink, M., Van Der Meulen, A., and Bierma-Zeinstra, S. M. (2004). The efficacy of Tai Chi Chuan in older adults: a systematic review. Fam. Pract. 21, 107-113. doi: 10.1093/fampra/cmh122

Virtanen, M., Elovainio, M., Josefsson, K., Batty, G. D., Singh-Manoux, A., and Kivimaki, M. (2017). Coronary heart disease and risk factors as predictors of trajectories of psychological distress from midlife to old age. Heart 103, 659-665. doi: 10.1136/heartjnl-2016-310207

Watkins, L. L., Blumenthal, J. A., Davidson, J. R., Babyak, M. A., Mccants, C. B. Jr., and Sketch, M. H. Jr. (2006). Phobic anxiety, depression, and risk of ventricular arrhythmias in patients with coronary heart disease. Psychosom. Med. 68, 651-656. doi: 10.1097/01.psy.0000228342. 53606.b3

Xiaolan, X. (2017). Effect of rehabilitation training combined with psychological intervention on cognitive function and mental behavior of dementia patients. J. Clin. Nurs. Res. 1, 51-55. doi: 10.26689/jcnr.v1i1.28

Xu, N., Meng, H., Liu, T., Feng, Y., Qi, Y., Zhang, D., et al. (2017). Blueberry phenolics reduce gastrointestinal infection of patients with cerebral venous thrombosis by improving depressant-induced autoimmune disorder via mir155-mediated brain-derived neurotrophic factor. Front. Pharmacol. 8:853. doi: 10.3389/fphar.2017.00853 
Zhang, X., Yoon, J. Y., Morley, M., Mclendon, J. M., Mapuskar, K. A., Gutmann, R., et al. (2018). A common variant alters SCN5A-miR-24 interaction and associates with heart failure mortality. J. Clin. Invest. 128, 1154-1163. doi: 10.1172/JCI95710

Zhao, Y., and Srivastava, D. (2007). A developmental view of microRNA function. Trends Biochem. Sci. 32, 189-197. doi: 10.1016/j.tibs.2007. 02.006

Zheng, G., Lan, X., Li, M., Ling, K., Lin, H., Chen, L., et al. (2014). The effectiveness of Tai Chi on the physical and psychological well-being of college students: a study protocol for a randomized controlled trial. Trials 15:129. doi: 10.1186/ 1745-6215-15-129

Zheng, G., Lan, X., Li, M., Ling, K., Lin, H., Chen, L., et al. (2015). Effectiveness of tai chi on physical and psychological health of college students: results of a randomized controlled trial. PLoS One 10:e0132605. doi: 10.1371/journal.pone. 0132605
Zheng, S., Kim, C., Lal, S., Meier, P., Sibbritt, D., and Zaslawski, C. (2018). The Effects of twelve weeks of tai chi practice on anxiety in stressed but healthy people compared to exercise and wait-list groups-a randomized controlled trial. J. Clin. Psychol. 74, 83-92. doi: 10.1002/jclp.22482

Conflict of Interest Statement: The authors declare that the research was conducted in the absence of any commercial or financial relationships that could be construed as a potential conflict of interest.

Copyright (C) $2019 \mathrm{Li}$, Zhang and Wang. This is an open-access article distributed under the terms of the Creative Commons Attribution License (CC BY). The use, distribution or reproduction in other forums is permitted, provided the original author(s) and the copyright owner(s) are credited and that the original publication in this journal is cited, in accordance with accepted academic practice. No use, distribution or reproduction is permitted which does not comply with these terms. 